\title{
Augmenting drug discussions in general practice
}

\author{
Robin De Croon \\ Department of Computer Science, KU Leuven \\ Celestijnenlaan 200A \\ BE-3000 Leuven \\ Email: robin.decroon@cs.kuleuven.be
}

\begin{abstract}
This extended abstract discusses our ongoing research on augmenting the communication between an expert and a layman using interactive visualizations. We are currently designing an interactive table that assists patients and general practitioners (GPs) to explore drug information using a camera to recognize drug packages and a projector to visualize complex drug information directly around these packages.
\end{abstract}

\section{INTRODUCTION}

An often stated solution to the challenges of healthcare such as keeping healthcare affordable and accessible is the 'empowered patient'. In order to empower the patient, information is a necessity. However, the data available is not always medically validated and sometimes misleading for the patient. Furthermore, it is not always straightforward to communicate complex health information to patients. We want to augment the communication between general practitioners (GPs) and their patients using interactive visualizations. Schneiderman, Plaisant and Hesse [1] discuss several opportunities where visualizations can help to improve healthcare. Based on requirements extracted from several interviews with patients, we will design interactive visualizations that assist GPs and patients to discuss complex drug information. More specifically, we are designing an interactive table that recognizes drug packages using computer vision techniques and that visualizes drug interactions, adverse drug reactions, intolerances and posology using a projector. A specific novelty of what we propose is the focus on improving the communication between an expert user (the GP) and a layman (the patient) using projected interactive visualizations with tangible objects in order to help patients gain insights about their drug use. We make use of tabletop affordances such as 'co-location', 'multiple users', 'hands-on activities' and 'multiple modes of communication' [2].

\section{RESEARCH QUESTIONS}

In our research, we want to learn in general 1) how we can improve the communication between an expert and a layman using interactive visualizations and, more specifically 2) how we can leverage tabletop affordances using information visualization techniques to augment adverse drug events awareness amongst patients. It should also be researched how medically validated data should be visualized in order to expose insights in the patient's health status.

\section{RESOURCES AND METHODS}

In our earlier research [3], [4] we intensively worked with GPs to design and evaluate designs that augment GPs' workflow. Furthermore, a collaborative funded project with industry partners enables us to get access to real data, real patients and real GPs - though our earlier experience makes it clear that availability of GPs for evaluation studies is always an issue. Finally, this research is situated in the augment group of the KU Leuven ${ }^{1}$ which provides us with the necessary expertise on human augmentation and information visualization.

We follow a user centered design methodology, where interviews with GPs and patients enable us to ground our research in real usage context. This means that users need to be able to touch the buttons of a working, realistic proof-of-concept and see how the visualizations actually work. With the help of proven methods such as structured interviews, think aloud user tests, task analysis and user tracking, we can update and enhance the prototypes in each iteration. In order to prevent that usability issues would influence our results, the usability of each prototype is measured with the system usability scale (SUS). The final evaluations rely on scenario-based evaluations [5].

\section{EXPECTED RESULTS}

After the rapid prototyping process, we expect to have a design which integrates our interactive visualizations and which augments the discussion about drug use between a GP and a patient. We thus hope to empower patients by raising their understanding of drug usage and reactions between drugs. In addition, thanks to using medically validated data, we hope to prevent known adverse drug events.

\section{REFERENCES}

[1] B. Shneiderman, C. Plaisant, and B. W. Hesse, "Improving Healthcare with Interactive Visualization," Computer, vol. 46, no. 5, pp. 58-66, May 2013.

[2] P. Dillenbourg and M. Evans, "Interactive tabletops in education," International Journal of Computer-Supported Collaborative Learning, vol. 6, no. 4, pp. 491-514, Dec. 2011.

[3] R. De Croon, J. Klerkx, and E. Duval, "Designing a useful and usable mobile emr application through a participatory design methodology: A case study," in Healthcare Informatics (ICHI), 2014 IEEE International Conference on, Sept 2014, pp. 176-185.

[4] - "Design and evaluation of an interactive proof-of-concept dashboard for general practitioners," in Healthcare Informatics (ICHI), 2015 IEEE International Conference on, Oct 2015, (in press).

[5] H. Lam, E. Bertini, P. Isenberg, C. Plaisant, and S. Carpendale, "Empirical Studies in Information Visualization: Seven Scenarios." IEEE transactions on visualization and computer graphics, vol. 18, no. 9, pp. 1520-1536, Nov. 2011.

${ }^{1}$ http://augment.cs.kuleuven.be 